\title{
Enhanced membrane protein expression by engineering increased intracellular membrane production
}

Mouna Guerfal ${ }^{1,2+}$, Katrien Claes ${ }^{1,2 \dagger}$, Oskar Knittelfelder ${ }^{3}$, Riet De Rycke ${ }^{4,5}$, Sepp D Kohlwein ${ }^{3}$ and Nico Callewaert ${ }^{1,2^{*}}$

\begin{abstract}
Background: Membrane protein research is frequently hampered by the low natural abundance of these proteins in cells and typically relies on recombinant gene expression. Different expression systems, like mammalian cells, insect cells, bacteria and yeast are being used, but very few research efforts have been directed towards specific host cell customization for enhanced expression of membrane proteins. Here we show that by increasing the intracellular membrane production by interfering with a key enzymatic step of lipid synthesis, enhanced expression of membrane proteins in yeast is achieved.
\end{abstract}

Results: We engineered the oleotrophic yeast, Yarrowia lipolytica, by deleting the phosphatidic acid phosphatase, $P A H 1$, which led to massive proliferation of endoplasmic reticulum (ER) membranes. For all eight tested representatives of different integral membrane protein families, we obtained enhanced protein accumulation levels and in some cases enhanced proteolytic integrity in the $\Delta p a h 1$ strain. We analysed the adenosine $A_{2 A} R$ G-protein coupled receptor case in more detail and found that concomitant induction of the unfolded protein response in the spah1 strain enhanced the specific ligand binding activity of the receptor. These data indicate an improved quality control mechanism for membrane proteins accumulating in yeast cells with proliferated ER.

Conclusions: We conclude that redirecting the metabolic flux of fatty acids away from triacylglycerol- and sterylester-storage towards membrane phospholipid synthesis by PAH1 gene inactivation, provides a valuable approach to enhance eukaryotic membrane protein production. Complementary to this improvement in membrane protein quantity, UPR co-induction further enhances the quality of the membrane protein in terms of its proper folding and biological activity. Importantly, since these pathways are conserved in all eukaryotes, it will be of interest to investigate similar engineering approaches in other cell types of biotechnological interest, such as insect cells and mammalian cells.

\section{Background}

Membrane proteins play critically important roles in a huge diversity of physiological processes. However, structural information on these proteins is scarce because of the multitude of experimental problems that need to be overcome to obtain a sufficient quantity of a detergentsolubilized, stable, homogeneous and monodisperse protein

\footnotetext{
*Correspondence: Nico.Callewaert@dmbr.vib-Ugent.be

${ }^{\dagger}$ Equal contributors

'Department for Molecular Biomedical Research, Unit for Medical Biotechnology, VIB, Technologiepark 927, 9052, Ghent, Belgium

${ }^{2}$ Department of Biochemistry and Microbiology, Laboratory for Protein Biochemistry and Biomolecular Engineering, Ghent University, KL.-Ledeganckstraat 35, 9000, Ghent, Belgium

Full list of author information is available at the end of the article
}

preparation from which well-ordered crystals for X-ray structural analysis can be grown. Only few membrane proteins have a natural abundance that is high enough to warrant purification from their native source, and heterologous overexpression is therefore required in most other cases. The expression systems that have been successfully used to produce membrane proteins for structure determination include mammalian cell lines, insect cells, bacterial cells and yeast. However, few expression systems have been specifically designed for the purpose of producing integral membrane proteins [1]. In part for that reason, only a small minority of membrane proteins can be readily overexpressed in a biologically active form, and this remains one of the bottlenecks on the way to a more

\section{Biomed Central}


robust workflow for membrane protein structure-function analysis. We here report on a eukaryotic endomembrane synthesis manipulation strategy for the overproduction of membrane proteins.

As membrane proteins accumulate in the host cell's intracellular and plasma membranes, we hypothesized that by providing a larger cellular membrane surface area the capacity to accommodate the overexpressed protein would increase. Many eukaryotic cells can absorb longchain fatty acids (FA) and store them in cytoplasmic lipid droplets in the form of triacylglycerols (TAG) and steryl esters (SE) [2]. The uptake of fatty acids from the culture medium can also directly provide the precursors necessary for phospholipid synthesis and could facilitate the biogenesis of membranes, if their incorporation into TAG and SE lipid stores could be suppressed. Thus, our attention was drawn to $P A H 1$, which encodes phosphatidic acid phosphatase Pah1p and which is a gate keeper for directing the flux of FA into TAG [3]. In PAH1deleted Saccharomyces cerevisiae, phosphatidic acid (PA) cannot be converted to diacylglycerol (DAG) and, consequently, no TAG can be made via the Pah1 pathway (Figure 1). Informed by the massive ER/nuclear membrane proliferation seen in a $\Delta p a h 1$ strain of $S$. cerevisiae [4] we investigated whether we could utilize this $\Delta p a h 1$ phenotype to enhance membrane protein productivity. We chose to work with Yarrowia lipolytica because fatty acid-regulated promoters are well established for this organism [5] and we hypothesized that the ability to feed direct precursors for membrane lipids (i.e. fatty acids) would be most suitable to achieve enhanced membrane lipid biosynthesis.

As our previously reported data in Pichia pastoris indicated that co-induction of the unfolded protein response (UPR) can enhance membrane protein homogeneity and activity [8], we also evaluated the combined

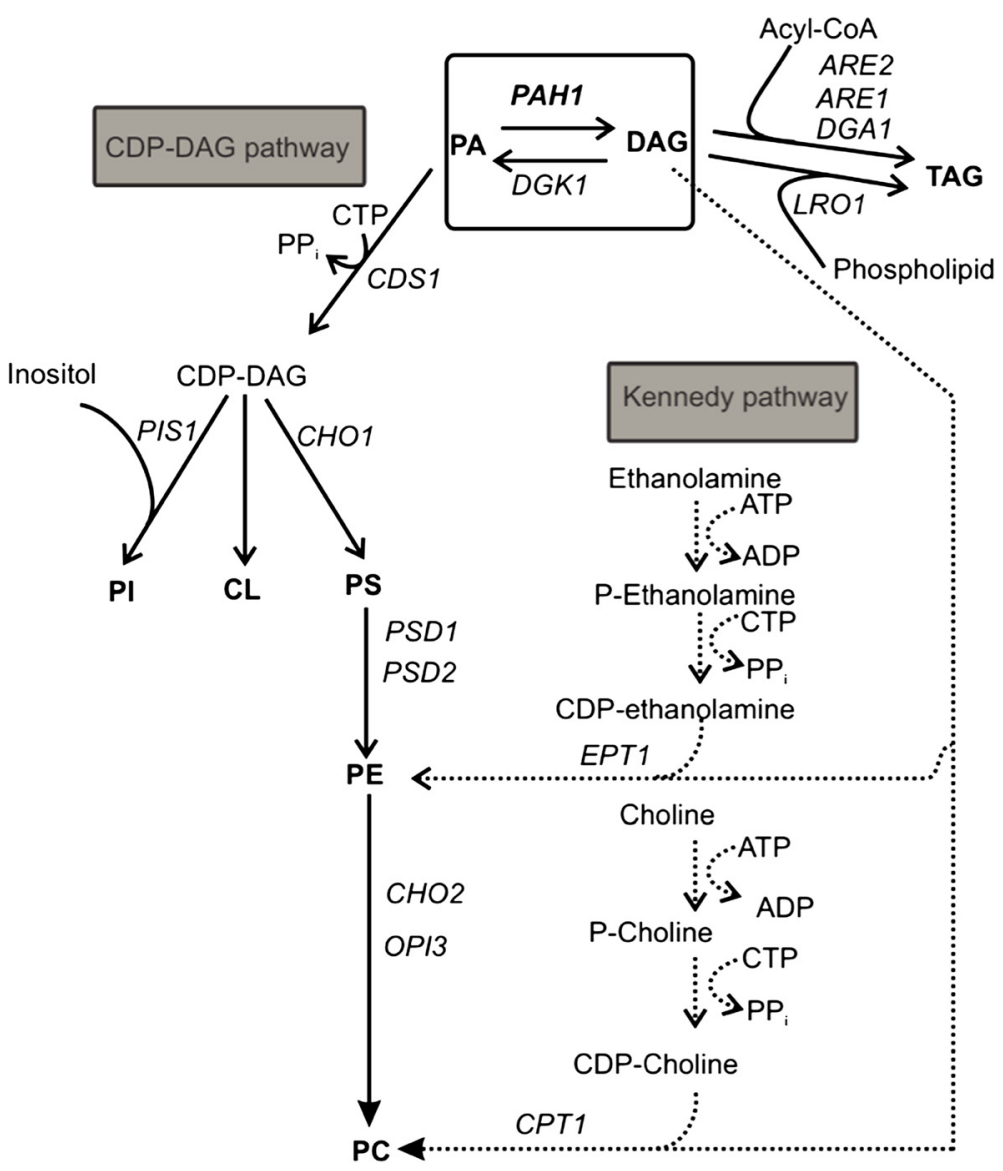

Figure 1 Schematic presentation of the major steps in phospholipid biosynthesis in yeast [6]. Phosphatidic acid phosphatase, encoded by PAH1, catalyzes the dephosphorylation of PA to DAG, the precursor of the storage lipid TAG (there are 3 more genes coding for enzymes with this activity in yeast, but only PAH1 is involved in the synthesis of TAG and the regulation of phospholipid synthesis [7]). All phospholipids can be derived from PA, with the exception of phosphatidylinositol which needs exogenous inositol input. PA, phosphatidic acid; DAG, diacylglycerol; TAG, triacylglycerol; PI, phosphatidylinositol; CL, cardiolipin; PS, phosphatidyl-serine; PE, phosphatidylethanolamine; PC, phosphatidylcholine. 
impact of endoplasmic reticulum (ER) membrane proliferation and UPR induction on the quantity and quality of the heterologously expressed membrane proteins.

\section{Results \\ Identification and knockout of the Yarrowia lipolytica PAH1 gene}

The sequence of the PAH1 gene of $Y$. lipolytica was identified through a homology search with the Saccharomyces cerevisiae PAH1 gene (GeneID: 855201) against the genome of $Y$. lipolytica, using the Basic Local Alignment Search Tool (Blast) from NCBI. The Blast analysis identified the protein YALI0_D27016p, which shows some similarities to the nuclear elongation and deformation protein 1 of Schizosaccharomyces pombe, the S. pombe orthologue of Pah1p [9]. The sequence contains the conserved HAD-like domain with a DxDxT motif, in the middle of the protein sequence [10]. The familycharacteristic N-terminal lipin domain was also identified (Additional file 1: Figure S1A).

A knockout construct for $P A H 1$ was made (Additional file 1: Figure $\mathrm{S} 1 \mathrm{~B})$ to replace the $P A H 1$ coding sequence by the sequence of the LEU2 selection marker [11]. The knockout was confirmed by PCR on genomic DNA (Additional file 1: Figure S1C).

\section{Impact of PAH1 deletion on Yarrowia lipolytica growth}

As our purpose was to use the $\Delta p a h 1$ strain for the large-scale production of membrane proteins, it was important to evaluate the impact of the $\Delta p a h 1$ mutation on the growth characteristics of the strain on glucose and oleic acid, the two carbon sources used for protein production with $Y$. lipolytica. Growth curves for the wild type and the $\Delta p a h 1$ strain were generated. Compared to the wild type control, growth of the mutant strain was only slightly retarded on glucose, and only during the early exponential phase (Figure 2A). Cells grown on oleic acid went through a longer lag phase but reached the stationary phase at the same optical density as the wild type strain (Figure 2B). These results show that the mutation does not appear to have a major impact on overall cellular physiology and does not present a serious obstacle to the utility of the strain for recombinant protein production.

\section{Membrane protein expression in the $\triangle p a h 1$ and $\Delta p a h 1 /$ Hac1p strain}

To begin to evaluate the impact of this lipid synthesis engineering step on the obtainable expression levels of heterologous integral membrane proteins, we chose to study the Adenosine $A_{2 A}$ receptor $\left(A_{2 A} R\right)$ that belongs to the pharmacologically highly interesting family of $\mathrm{G}$ protein coupled receptors (GPCRs). Radioligand binding assays are available to evaluate the functionality of the protein [12]. First, we generated a wild type $Y$. lipolytica strain expressing the $A_{2 A} R$ from a single integrated gene copy under control of the oleic acid-inducible POX2 promoter. We then generated a $\Delta p a h 1$ derivative of this strain, ensuring that the wild type and $\Delta p a h 1$ strains expressed the $\mathrm{A}_{2 \mathrm{~A}} \mathrm{R}$ in the same genetic background.

Furthermore, we studied whether this $\Delta p a h 1$ membrane capacity engineering could be usefully integrated with a concomitant enhancement of the ER protein quality control capacity as afforded by induction of the unfolded protein response. The UPR in yeast is triggered by non-canonical splicing of the HAC1 mRNA [13]. We identified the splice event in $Y$. lipolytica (as also identified since in [14]) and generated an expression construct for the spliced active Yarrowia $H A C 1$, driven by the POX2 promoter. Subsequently, both the wild type and the $\Delta p a h 1 \mathrm{~A}_{2 \mathrm{~A}} \mathrm{R}$-expressing strains were transformed with this expression vector.

The expression levels of $\mathrm{A}_{2 \mathrm{~A}} \mathrm{R}$ in the different strains were determined by western blot analysis (Figure $3 \mathrm{~A}$ ).
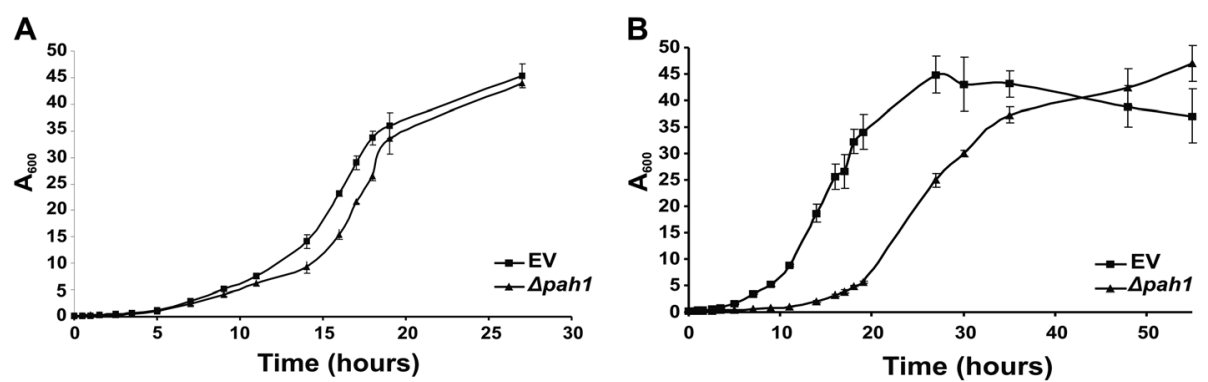

Figure 2 Evaluation of the $\Delta p a h 1$ growth phenotype. The growth phenotype of an empty vector strain and the $\Delta p a h 1$ strain was compared on glucose and oleic acid as carbon sources. The empty vector strain (EV) is the PO1d $\triangle O C H 1$ strain transformed with a plasmid containing the LEU selection marker cassette to rule out any effect of the selection pressure, compared to the PAH1 knockout strain ( $\triangle p a h 1)$, which carries the LEU selection marker on the knockout construct. A. Growth on glucose as carbon source for 27 hours. Growth of the knockout was retarded only slightly and in the stationary phase, cells reached the same $\mathrm{OD}_{600}$ as the wild type strain. B. Growth on oleic acid as carbon source for 55 hours. Also here, the PAH1 knockout strain has a prolonged lag phase but it reaches the stationary phase at the same $\mathrm{OD}_{600}$ as the empty vector strain. 

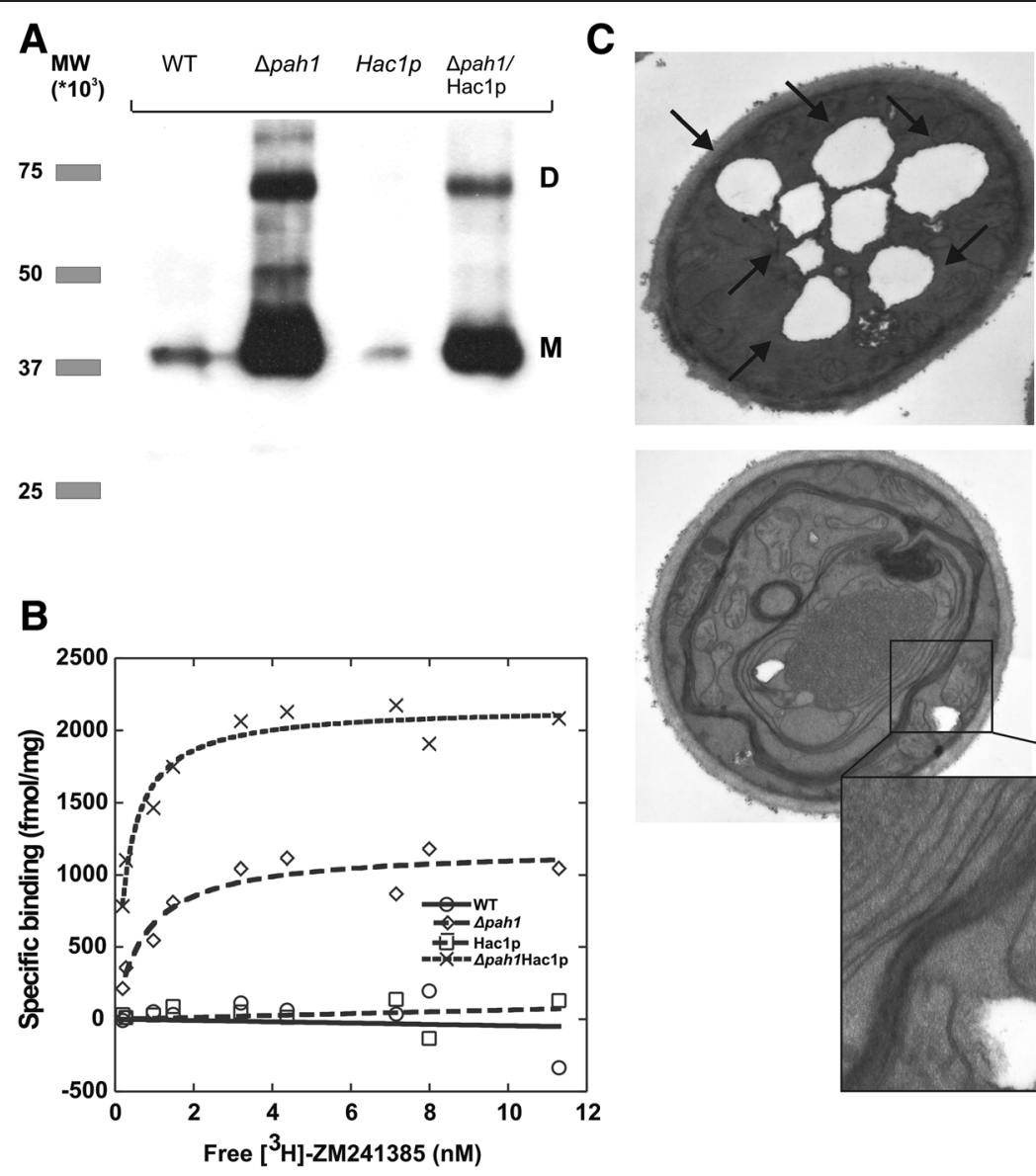

WT

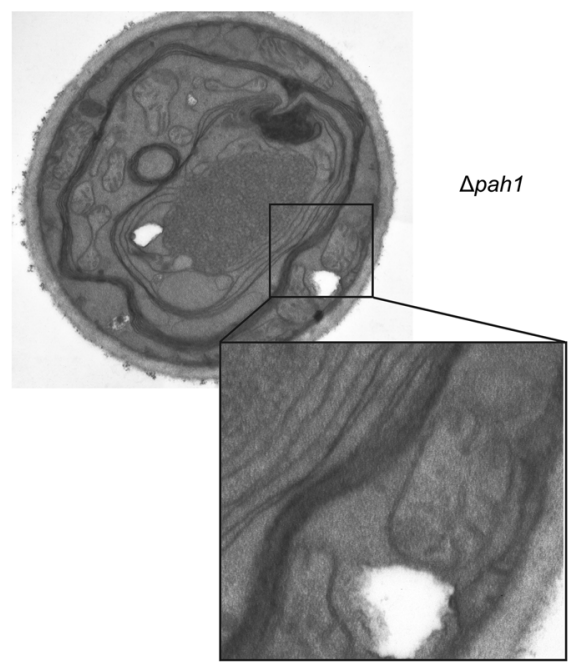

D

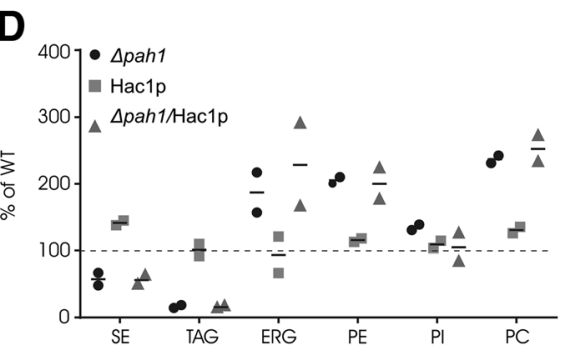

Figure 3 Analysis of $A_{2 A} R$ expression and membrane expansion in the $4 p a h 1$ strain. $\mathbf{A}$. Western blot analysis of $A_{2 A} R$ in $10 \mu g$ of total membrane proteins of wild type, $\triangle p a h 1, H A C 1$ co-overexpression and $\triangle p a h 1 / H a c 1 p$ strains. B. Radioligand binding studies on membranes expressing the $A_{2 A} R$. Deletion of the PAH1 gene leads to a strong increase (over 20-fold) in production of ligand-binding receptor. Artificial induction of the UPR reduces the quantity of receptor produced, but strongly enhances the radioligand binding activity, indicating enhanced receptor protein quality. C. EM pictures were taken after 48 hours growth on oleic acid of both wild type and $\Delta p a h 1$ cells. When the knockout cells are grown on oleic acid, whirls and stacked layers of proliferated membrane become apparent. Also, few or no lipid droplets are present in the knockout strain, while big droplets are present in the wild type cells (arrows). D. HPLC-LSD analysis of the major cellular lipid classes derived from the strains grown on oleic acid. Lipid levels were quantified relative to those in the wild type parental strain (represented as 100\%, broken horizontal line). The values derived from two independent experiments are shown, with their average represented by the short horizontal lines.

The results demonstrate that the $\Delta p a h 1$ mutation very strongly enhanced the expression levels of the GPCR. qPCR analysis showed that the mRNA levels for the GPCR were only slightly enhanced in the $\Delta p a h 1$ strain (non-significant, $\mathrm{P}=0.083$, Mann-Whitney U-test, $\mathrm{n}=8$ ) (Additional file 2: Figure S2), indicating that stronger promoter activation and/or altered mRNA metabolism in the knockout is not the main causative factor for the strongly enhanced membrane protein accumulation. The $\Delta p a h 1 /$ Hac1p combination strain showed a somewhat lower production yield of the $\mathrm{A}_{2 \mathrm{~A}}$ receptor than the $\Delta$ pah1 strain (Figure $3 \mathrm{~A}$ ). We next performed $\mathrm{A}_{2 \mathrm{~A}} \mathrm{R}$ ligand binding studies on total membrane protein extracts to study whether the enhanced receptor expression levels 
also correlated with enhanced levels of ligand bindingcompetent receptor (Figure $3 \mathrm{~B}$ ). Under our test conditions, no binding of the ligand was detectable in the wild type POX2- $\mathrm{A}_{2 \mathrm{~A}} \mathrm{R}$ strain, whereas in the derived $\Delta p a h 1$ strain, $B_{\max }$ was $1178 \pm 81 \mathrm{fmol}$ per mg of total membrane protein. The lack of detectable ligand binding in the wild type strain was most likely due to the very low expression level of the receptor or its poor folding, or both.

Although the protein expression levels were similar in the $\Delta p a h 1$ and $\Delta p a h 1 / \mathrm{Hac} 1 \mathrm{p}$ combination strains, we observed almost 2 -fold increased specific radioligand binding activity of the receptor when Hac1p was co-overexpressed $(2167 \pm 65 \mathrm{fmol}$ per $\mathrm{mg}$ of total membrane protein). We speculate that this is due to enhanced ER-chaperone mediated folding. In addition, enhanced ER associated degradation (ERAD) may aid in degrading molecules that are not folded in a ligand-binding competent state.

We also assessed intracellular membrane morphology in the wild type and $\Delta p a h 1$ strains by electron microscopy (EM) of cells cultivated to high cell density (which is relevant for heterologous protein production) on oleic acid (Figure $3 \mathrm{C}$ ). Wild type cells growing on oleic acid massively overproduce TAG, which is stored in cytosolic lipid droplets. In stark contrast, EM analysis of the $\Delta p a h 1$ strain revealed massive intracellular membrane proliferation and almost complete absence of lipid droplets. This observation is consistent with the metabolic function of Pah1p to regulate the flux of phosphatidic acid into TAG synthesis.

The enhanced lipid fluxes towards membrane lipids in the $\Delta p a h 1 Y$. lipolytica strain grown on oleic acid were confirmed by HPLC-light scattering detection analysis of cellular lipid content and composition (Figure 3D): in the $\Delta p a h 1$ mutant, the TAG pool was reduced approximately 5 -fold, and the SE pool was reduced about 2 -fold as compared to the wild type strain, whereas the levels of the membrane lipids ergosterol (ERG), phosphatidylethanolamine (PE) and phosphatidylcholine (PC) were all increased about 2-fold. The levels of phosphatidylinositol were similar to the wild type strain. $H A C 1$ overexpression in the $\triangle p a h 1$ background did not alter this lipid composition, excluding the possibility that the HAC1 overexpression effect on membrane protein quality was due to membrane lipid alterations (Figure 3D).

\section{The $\Delta$ pah1 mediated membrane protein overexpression is broadly applicable}

To explore whether the massive intracellular membrane expansion seen in the $\Delta p a h 1$ strain was specific to $\mathrm{A}_{2 \mathrm{~A}} \mathrm{R}$ or had a beneficial effect on membrane protein yield and function in general, we studied expression of seven additional integral membrane proteins: the human $5 \mathrm{HT}_{1 \mathrm{D}}$ receptor, human mu opioid receptor, aquaporin Aqy1 of Pichia pastoris, human B-cell associated receptor 31, porcine respiratory virus (PRV) NS4, presenilin/SPP homologue of Methanoculleus marisnigri JR1, and human cytochrome P450 2D6. For all of these proteins, as for the $\mathrm{A}_{2 \mathrm{~A}} \mathrm{R}$ described above, we first generated expressing clones and then knocked out PAH1 in all of these strains. While significantly expanding the effort, this approach ascertains that differences in protein expression between the wild type and the mutants are not due to e.g. differences in transgene insertion site or copy number. All of the expressed membrane proteins in our analysis show a moderate to strong increase in the expression level in the $\Delta p a h 1$ strain. In cases where proteolysis occurred in the wild type strain, this was much less the case in the $\Delta p a h 1$ strains, for example for Bap31 and PRV NS4 (Figure 4). Thus, we conclude that deletion of the PAH1 gene and enhanced membrane production in the presence of oleate provides a viable and widely applicable strategy for membrane protein expression.

\section{Discussion}

In the last few years, several breakthroughs have been achieved in the ability to stabilize membrane proteins (especially GPCRs) by engineering the molecules themselves; these strategies have yielded several crystal structures of those membrane proteins that express relatively well in standard expression systems $[15,16]$. Together with

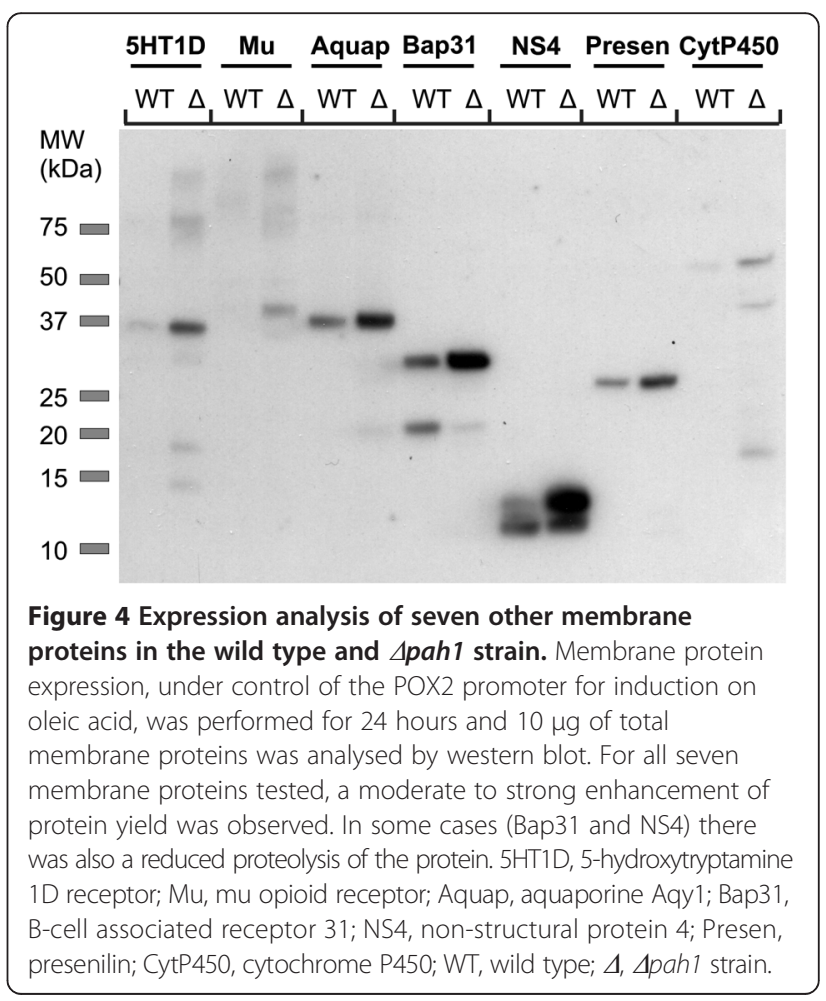


these efforts, we expect that custom-engineered expression systems for integral membrane proteins, such as described here, will allow access to a much broader spectrum of membrane proteins than has been possible to date.

In the $\Delta p a h 1$ strain, the cells are severely impaired in neutral lipid synthesis and consequently showed a strong reduction or absence of lipid droplets, which was also reported for S. cerevisiae [3]. The absence of this lipid droplet sink for fatty acids, makes the strain potentially susceptible to fatty-acid induced lipotoxicity. Indeed, the mutant strain showed a somewhat extended lag phase compared to wild type cells, however, it readily adapted to the presence of excess oleic acid and grew to similar cell densities as the wild type strain, in stationary phase. Although $S$. cerevisiae strains defective in neutral lipid synthesis (Dare1 $\Delta a r e 2 \Delta d g a 1 \Delta$ lro1) also show an extended lag phase on oleic acid and eventually reach wild type levels of cell density [17], this adaptation is likely due to second site suppressors [18]. Thus, Yarrowia - an oleaginous yeast - is much more tolerant to alterations in fatty acid fluxes. In any case, since a similar culture density is obtained with the Yarrowia $\Delta p a h 1$ strain as with the wild type, this strain is entirely suitable for biotechnological protein production. All work reported here is based on simple shake flask cultivation with singlecopy strains, thus, further strategies to enhance the yield of the strains developed in this study include optimal oleic acid feeding in controlled bio-fermentors, and exploring the effect of gene copy number increases. In addition, newer generations of stronger promoters for Yarrowia have recently been described $[19,20]$ and will be useful to enhance the obtainable expression levels.

Importantly, the unfolded protein response and the master lipid flux regulatory role of Pah1p orthologues (lipins) [21], are conserved in all eukaryotes, including insect cells and mammalian cells. Technology to knock down or knock out genes in these organisms is now readily available [22,23]. Therefore, there is scope for applying the same membrane engineering approach to these other frequently used (membrane) protein expression hosts. However, careful exploration of an appropriate match between the carbon source and the promoter systems will be required for other biotech cell types in which the $\Delta p a h 1$ manipulation is attempted and this will be the subject of further studies. For example, while Pichia pastoris is widely used for heterologous protein expression and extremely strong methanol-inducible promoters are available, our preliminary data (unpublished) suggest that C1-metabolism is not compatible with $\Delta$ pah1-mediated membrane expansion: the membranes appear to be autophagocytosed, obliterating any beneficial effect on membrane protein expression yields. However, the rapidly expanding genetic toolbox for Pichia [24] will allow to match appropriate promoter systems with carbon source feeding strategies that allow for $\Delta p a h 1$-mediated stable membrane surface expansion.

\section{Conclusions}

We conclude that $\Delta p a h 1$-mediated redirection of lipid synthesis fluxes away from storage lipids and towards membrane lipids, leads to a strong intracellular membrane proliferation especially when the cells are fed with fatty acids. This membrane expansion affords enhanced expression levels and proteolytic integrity of integral membrane proteins. We furthermore conclude that coinduction of the UPR in such $\triangle p a h 1$ strains improves the quality of the overproduced membrane proteins. The engineered pathways are conserved in all eukaryotes, which offers a scope for application in other yeasts, insect cells and mammalian cells. To our knowledge, this is the first successful attempt to engineer the membrane synthesis machinery of a eukaryotic expression system to customize cells for accommodating integral membrane proteins. The improved yield, stability and activity of membrane proteins in these mutant strains are likely to enhance progress on their functional and structural characterization.

\section{Methods}

\section{Strains}

E. coli MC1061 was used for standard molecular biology manipulations. E. coli was grown in LB medium supplemented with the appropriate selection antibiotics. Yarrowia lipolytica PO1d $\triangle O C H 1$ was used for analysis and protein expression. This strain is a knockout for the $\mathrm{OCH} 1$ mannosyltransferase in the wild type PO1d strain (CLIB 139), which results in the absence of $\mathrm{N}$-glycan hypermannosylation, which is beneficial for more homogenous glycoprotein production [25].

\section{Transformation}

Transformation was performed by the lithium acetate method as described by Barth and Gaillardin [26].

\section{Generation and identification of a $\mathrm{PAH} 1$ knockout strain}

A PAH1 knockout strain was generated according to the protocol described by Fickers et al. [11] and as depicted in Additional file 1: Figure S1B. Genomic DNA was isolated using the MasterPureTM Yeast DNA Purification Kit according to the manufacturer's instructions (Epicentre Biotechnologies, WI, USA). Clones that were prototrophic for leucine were then genotyped by PCR with primers pah1ylPfw07-010 (5'-GCGGCCGCGAAGACGGTGAGTATGGCCATC-3') and pah1ylTrv07-007 (5' -GCGGCC GCCCAAACCATGCATACAAATCAG- 3'. PCR was performed with Phusion polymerase (Thermo Scientific, MA, USA): $5 \mathrm{~min}$ at $98^{\circ} \mathrm{C}$, followed by 25 cycles of 1 min at $98^{\circ} \mathrm{C}, 20 \mathrm{sec}$ at $60^{\circ} \mathrm{C}$, and $45 \mathrm{sec}$ at $72^{\circ} \mathrm{C}$. A band 
of 3268 bp was expected for the wild type PAH1 and a band of $2966 \mathrm{bp}$ for the $\Delta p a h 1$ strain. Random integration would lead to the presence of both bands.

\section{Cloning of spliced form of Y. lipolytica HAC1 and construction of Hac1p expression plasmid}

The spliced form of HAC1 mRNA from Y. lipolytica PO1d was isolated as described earlier [8]. In brief, UPR was induced by adding DTT to $5 \mathrm{mM}$ to an exponentially growing culture. RNA was isolated and reverse transcribed as described. The spliced cDNA was cloned into the BamHI/AvrII sites in plasmid JMP62hyg, which is a Yarrowia expression vector containing the POX2 promoter and a hygromycin resistance cassette. The resulting plasmid is called JMP62hygHAC1(s). The plasmid was cut with $K p n I$ before transformation to allow integration in the POX2 promoter locus.

Construction of the membrane protein expression vectors The coding sequence of the Adenosine $\mathrm{A}_{2 \mathrm{~A}}$ receptor was isolated from a fetal brain cDNA library with primers 5' -GAATGCAATGCCCATCATGGGCTCC-3' and $5^{\prime}$-CCTAGGTCAAGCTGGAGCAACTTGAGAAG3', which include the BsmI and AvrII restriction sites. The isolated gene was cloned in the $\mathrm{pCR}^{\circ}$-Blunt II-TOPO vector (Invitrogen). The insert in the resulting vector $\mathrm{pTO}$ POA2A was sequence-verified. The open reading frame was then cloned in the vector pYLPUXdl2prepro, which contains a ura3d1 selection marker, the POX2 promoter and the secretion signal of the LIP2 gene for fusion to the coding sequence of the protein of interest. To obtain the construct expressing the $\mathrm{A}_{2 \mathrm{~A}}$ receptor from the POX2 promoter, this vector was cut with FseI and AvrII. The coding sequence of the $\mathrm{A}_{2 \mathrm{~A}}$ receptor was isolated from the pTOPOA2A vector by $B s m \mathrm{I} / A v r I I$ digestion. The appropriate fragments were ligated and the final plasmid was called pPOX2-A2A. The genes of the seven other membrane proteins, fused to the LIP2 secretion signal, were produced using synthetic DNA technology (GenScript, NJ, USA) with terminal BamHI/AvrII restriction sites for cloning into the pYLPUXdl2prepro backbone.

\section{Growth curve}

To analyse the growth phenotype of the PAH1 knockout strain, a comparison was made with an empty vector (EV) strain. This EV strain is the PO1d $\triangle O C H 1$ strain transformed with a NotI-linearized plasmid, pJMP62LEU, containing the LEU selection marker cassette, making this strain leucine-prototrophic, as the knockout strain. Cultures were grown overnight to saturation in YPD (1\% yeast extract, $2 \%$ peptone, $2 \%$ dextrose) or YTO (50 mM phosphate buffer $\mathrm{pH} 6.8,1 \%$ yeast extract, $2 \%$ tryptone, $2 \%$ oleic acid) and diluted the next day to an
$\mathrm{OD}_{600}$ of 0.2 . Incubation was continued and $\mathrm{OD}_{600}$ was measured periodically. For the cultures grown on medium containing oleic acid, the cells were spun down for 10 minutes at 13,000 rpm and washed once with $0.1 \mathrm{~N}$ $\mathrm{NaOH}$ to avoid interference of oleic acid in the $\mathrm{OD}_{600}$ measurement. Samples were measured in triplicate.

\section{Small scale expression}

Induction experiments for the Adenosine $\mathrm{A}_{2 \mathrm{~A}}$ receptor were performed in $12.5 \mathrm{ml}$ of culture medium in a $125-\mathrm{ml}$ baffled flask, for the other membrane proteins, induction was performed in $2 \mathrm{ml}$ of culture medium in 24 deep well plates. For all experiments, an overnight pre-culture was used to inoculate medium to an OD of 0.1 . Cells were grown for 24 hours in YTD (1\% yeast extract, $2 \%$ tryptone, $2 \%$ dextrose), washed once with water, and then resuspended in induction medium $(50 \mathrm{mM}$ phosphate buffer pH $6.8,1 \%$ yeast extract, $2 \%$ tryptone, $2 \%$ oleic acid). Cells were cultivated for another 24 hours before harvesting.

\section{Membrane protein preparation}

Cells (approximately $12 \times 10^{8}$ cells) were pelleted by centrifugation at $1,500 \times \mathrm{g}$ and the pellet was resuspended in ice-cold disruption buffer $(50 \mathrm{mM}$ sodium phosphate buffer $\mathrm{pH} 7.4$ supplemented with Complete protease inhibitor cocktail (Roche)). Cells were then broken by vigorous vortexing with glass beads in a mixer mill for $5 \times 2 \mathrm{~min}$ at $4^{\circ} \mathrm{C}$. Cell debris was separated from the membrane suspension by low speed centrifugation $(1,000 \times \mathrm{g}$, $\left.30 \mathrm{~min}, 4^{\circ} \mathrm{C}\right)$. Total cellular membranous organelles were pelleted at $100,000 \times \mathrm{g}$ at $4^{\circ} \mathrm{C}$ for $60 \mathrm{~min}$. For all membrane proteins analysed in Figure 4, the ultracentrifugation step was substituted for centrifugation at $13,000 \times \mathrm{g}$ at $4^{\circ} \mathrm{C}$ for $60 \mathrm{~min}$, as this was found not to be required to enable western blot analysis, and to yield very similar results as the protocol involving ultracentrifugation. The membranous pellet was resuspended in $50 \mathrm{mM}$ sodium phosphate buffer $\mathrm{pH} 7.4$ supplemented with Complete protease inhibitor cocktail and snap-frozen in liquid nitrogen until further testing. The protein concentration of the membrane preparation was determined using the BCA reagent (Pierce, Rockford, IL) with BSA as a standard. Ten micrograms of total membrane protein were analyzed by western blot. The blot was blocked overnight in $0.05 \%$ Tween-20 and 3\% casein in 1x PBS and probed with a $1 / 500$ diluted primary mouse anti-Rho1D4 antibody, followed by a 1/3000 diluted secondary antimouse IgG peroxidase from sheep (Sigma Cat. ${ }^{\circ}$ NA931V). Protein bands were visualized with Renaissance western blot Chemiluminescence Reagent Plus (PerkinElmer).

\section{Ligand binding of Adenosine $A_{2 A}$ receptor}

The procedures for studying binding to recombinant $\mathrm{A}_{2 \mathrm{~A}}$ receptors have been described [12]. Briefly, $10 \mu \mathrm{g}$ of 
total membrane proteins was incubated with different concentrations of the $\mathrm{A}_{2 \mathrm{~A}} \mathrm{R}$ antagonist, [3H]ZM241385 (0.05-12 $\mathrm{nM})$, in $500 \mu \mathrm{l}$ of binding buffer $(20 \mathrm{mM}$ HEPES pH 7.4, $100 \mathrm{mM} \mathrm{NaCl}$ ). Adenosine deaminase $(0.1 \mathrm{U})$ was added to degrade the adenosine released from the membranes, and the membranes were incubated at $22^{\circ} \mathrm{C}$ for 1 hour. Non-specific binding was determined in the presence of $10 \mathrm{mM}$ theophylline. Measurements were performed in duplicate. After incubation, bound and free ligands were separated on Whatmann GF/B filters pretreated with $0.1 \%$ polyethylenimine using a Brandel cell harvester. The filters were washed three times with binding buffer and the amount of bound radioligand was measured on a liquid scintillation counter. $\mathrm{Kd}$ and Bmax were determined by rectangular hyperbole curve fitting using KaleidaGraph software (Synergy Software).

\section{Electron microscopy}

Samples were prepared for EM according to Baharaeen et al. [27]. Yeast cells were fixed for $2 \mathrm{~h}$ on ice in $1.5 \%$ paraformaldehyde and $3 \%$ glutaraldehyde in $0.05 \mathrm{M}$ sodium cacodylate buffer, $\mathrm{pH}$ 7.2. After washing three times for $20 \mathrm{~min}$ in buffer, cells were treated with a $6 \%$ aqueous solution of potassium permanganate for $1 \mathrm{~h}$ at room temperature, to enhance membrane contrast. After washing three times for $20 \mathrm{~min}$ in buffer, cells were dehydrated through a graded ethanol series, including bulk staining with $2 \%$ uranyl acetate at the $50 \%$ ethanol step, followed by embedding in Spurr's resin. Ultrathin sections of a gold interference colour were cut using an ultra microtome (Ultracut E; Reichert-Jung), post-stained with uranyl acetate and lead citrate in a Leica ultrastainer, and then collected on formvarcoated copper slot grids. They were viewed with a transmission electron microscope 1010 (JEOL, Tokyo, Japan).

\section{Reverse transcription quantitative PCR}

Eight biological replicates of both the wild type strain and the PAH1 deletion strain were grown and induced as described above. After 24 hours of induction, 6.10E8 cells were pelleted and washed twice with $0.1 \mathrm{M} \mathrm{NaOH}$ at room temperature to completely remove oleic acid. Total RNA was prepared using the RiboPure yeast kit (Ambion, Life Technologies) according to the manufacturer's protocol. $10 \mu \mathrm{g}$ of total RNA was submitted to DNase treatment using the TURBO DNA-free kit (Ambion, Life Technologies). RNA integrity was confirmed for all sixteen samples using the Agilent RNA 6000 Pico Kit on the BioAnalyzer 2100 (Agilent Technologies). cDNA was prepared from $2 \mu \mathrm{g}$ DNase-treated RNA using the iScript Synthesis Kit (BioRad) and a control reaction lacking reverse transcriptase was included for each sample. The RT-PCR program was as follows: 10 minutes at $25^{\circ} \mathrm{C}, 30$ minutes at $42^{\circ} \mathrm{C}, 5$ minutes at $85^{\circ} \mathrm{C}$, and then cooling down to $12^{\circ} \mathrm{C}$.
Real time quantitative PCR was done on a LightCycler 480 (Roche Diagnostics) using the SensiFast SYBR-NoRox kit (BioLine), in triplicate for each RNA sample, on a 384-multiwell plate, with 5\% cDNA in a total volume of $10 \mu \mathrm{L}$. Primers were used at a final concentration of $10 \mu \mathrm{M}$. All primer pairs were generated using PrimerBlast (http://www.ncbi.nlm.nih.gov/tools/primer-blast) and verified for cross-specificity within the Yarrowia genome, as well as optimized for reduced secondary structure formation and reduced duplex formation. Primers used in the final experiment can be found in Additional file 3: Table S1. The following programme for cDNA synthesis was used: $3^{\prime}$ at $95^{\circ} \mathrm{C}$, and 45 times $3^{\prime \prime}$ at $95^{\circ} \mathrm{C}-30^{\prime \prime}$ at $60^{\circ} \mathrm{C}-1^{\prime \prime}$ at $72^{\circ} \mathrm{C}$. The necessary controls (no reverse transcriptase controls, no template controls) were included. The stability of 10 candidate reference genes for normalization was analyzed in a pilot experiment (data not shown) using the genormPLUS algorithm, as implemented in the qbasePLUS software [28]. Based on these results, all gene expression values were normalized using the geometric mean of the genes PGK (Gene ID: 2910137) and QCR9 (Gene ID: 2906637). Determination of amplification efficiencies and conversion of raw $\mathrm{Cq}$ values to normalized relative quantities (NRQ) were performed using the the qbasePLUS software [28]. Statistical analyses of NRQs were done with the Prism 6 software package using the Mann-Whitney U-test.

\section{Lipid extraction}

Lipid extraction of wild type, PAH1 knockout, Hac1p overexpressing and $\Delta p a h 1 / \mathrm{Haclp}$ strains was performed. After cultivation as described above, cells corresponding to $20 \mathrm{OD}_{600}$ units were harvested and disrupted with glass beads in chloroform/methanol 2:1 (v/v), by shaking in a Heidolph Multi Reax test tube shaker (Schwabach, Germany). Lipids were extracted according to Folch et al. [29].

\section{HPLC-LSD}

The chromatographic setup consisted of an Agilent 1100 combining pump, injector, precooled sample manager $\left(4^{\circ} \mathrm{C}\right)$ and column oven $\left(40^{\circ} \mathrm{C}\right)$. For detection of the lipids, a Sedex 85 evaporative light scattering detector (Sedere, France) was used. Data acquisition was performed by the Chemstation software (B 04.01). A ternary gradient (modified from [7]) with a Betasil Diol column $(100 \times 4.6 \mathrm{~mm}$, particle size $5 \mu \mathrm{m}$, Thermo $)$ was used to separate the various lipid classes. Neutral lipid standards were purchased from Larodan, except for ergosterol (Acros Organics) and cholesterylpalmitate (Sigma Aldrich). Neutral lipid and phospholipid (Avanti Polar Lipids) standards were prepared as $1 \mathrm{mg} / \mathrm{ml}$ stock solutions in chloroform/methanol 2:1 (v/v). Calibration curves 
were measured from $2.7 \mu \mathrm{g} / \mathrm{ml}$ to $350 \mu \mathrm{g} / \mathrm{ml}$. The injection volume for all calibration standards and samples was $10 \mu \mathrm{l}$.

\section{Additional files}

Additional file 1: Figure S1. Yarrowia lipolytica PAH1 gene and PAH1-knockout generation. A. Domain structure of the Pah1 protein, where the conserved $\mathrm{N}$-lipin domain is shown in green. The asterix represents the conserved glycine residue, which, together with the aspartic acid residues in the HAD domain (orange), is necessary for the phosphatidic acid phosphatase activity of Pah1p. B. Knockout strategy used to delete the PAH1 gene. Integration of the knockout cassette replaces the PAH1 gene by the LEU2 selection marker. The strategy for knocking out the PAH1 gene was set up as described in Fickers et al. [11]. We generated a construct that includes the promoter and terminator fragment of the PAH1 gene and a LEU2 marker for selection. A knockout is obtained after double homologous recombination at the promoter and terminator sites of PAH1. C. After transformation with the PAH1 knockout construct (P-LEU2-T), transformants were isolated and genotyped for the PAH1 gene locus. Lane 1, 1 kb DNA marker (Promega). Lanes 2 and 3, PCR amplificate of the PAH1 gene locus in, respectively, the wild type PO1d strain and an empty vector plasmid strain (expected wild type PAH1 amplificate size is $3268 \mathrm{bp}$ ). Lane 4, PCR amplificate of the disrupted PAH1 gene locus in a knockout strain (expected amplificate size is 2966 bp).

Additional file 2: Figure S2. Adenosine $A_{2} A$ transgene mRNA expression in the wild type and PAH1 deletion strains. Graph showing the normalized relative quantities of the adenosine $A_{2 A}$ receptor transgene mRNA in the wild type and PAH1 deletion strains. A trend towards more transgene mRNA in the knockout strain can be observed, which however did not reach statistical significance $(p=0.083)$, even with 8 biological replicates. (Mann-Whitney U-test). Horizontal bars represent median with the interquartile ranges.

Additional file 3: Table S1. GPCR primers of protein-coding genes.

\section{Competing interests}

The authors declare to have no competing financial interests.

\section{Authors' contributions}

MG and KC designed and performed experiments, analyzed and interpreted data and drafted the manuscript. RDR performed the EM analysis, OK conducted the HPLC-LSD measurements and interpreted the results, SDK supervised and interpreted the lipid analysis experiments. N.C. initiated and designed the study, coordinated the project, interpreted data and co-wrote the manuscript. All authors read and approved the final version of the manuscript.

\section{Acknowledgements}

MG and KC are supported by a Ph.D. grant from the Institute for the Advancement of Scientific and Technological Research in Industry (IWT-Vlaanderen). SDK is supported by the Austrian Science Fund (FWF), project F3005 LIPOTOX, and OK is supported by the Austrian Science Fund (FWF) PhD program 'Molecular Enzymology' (project W901-B05). Research in the lab of NC is supported by the Marie Curie Excellence Grant MEXT-014292 under EU Framework Program 6 and Research grant G.0.541.08.N.10 of the Fund for Scientific Research Flanders (FWO). We thank Dr. Wouter Vervecken for providing the expression plasmids.

\section{Author details}

'Department for Molecular Biomedical Research, Unit for Medical Biotechnology, VIB, Technologiepark 927, 9052, Ghent, Belgium. ${ }^{2}$ Department of Biochemistry and Microbiology, Laboratory for Protein Biochemistry and Biomolecular Engineering, Ghent University, K.L.-Ledeganckstraat 35, 9000, Ghent, Belgium. ${ }^{3}$ Institute of Molecular Biosciences, University of Graz, Humboldtstrasse 50/II, A8010 Graz, Austria. ${ }^{4}$ Department for Molecular Biomedical Research, Microscopy Core Facility, VIB, Technologiepark, 927, 9052 Ghent, Belgium. ${ }^{5}$ Department of Biomedical Molecular Biology, Ghent University, Technologiepark, 927, 9052 Ghent, Belgium.
Received: 14 August 2013 Accepted: 3 December 2013

Published: 9 December 2013

\section{References}

1. Zoonens M, Miroux B: Expression of membrane proteins at the Escherichia coli membrane for structural studies. Methods Mol Biol 2010, 601:49-66.

2. Rajakumari S, Grillitsch K, Daum G: Synthesis and turnover of non-polar lipids in yeast. Prog Lipid Res 2008, 47(3):157-171.

3. Pascual F, Carman GM: Phosphatidate phosphatase, a key regulator of lipid homeostasis. Biochim Biophys Acta 2013, 1831(3):514-522.

4. Santos-Rosa H, Leung J, Grimsey N, Peak-Chew S, Siniossoglou S: The yeast lipin Smp2 couples phospholipid biosynthesis to nuclear membrane growth. EMBO J 2005, 24:1931-1941.

5. Nicaud JM, Madzak C, van den Broek P, Gysler C, Duboc P, Niederberger P, Gaillardin C: Protein expression and secretion in the yeast Yarrowia lipolytica. FEMS Yeast Res 2002, 2(3):371-379.

6. Henry SA, Kohlwein SD, Carman GM: Metabolism and regulation of glycerolipids in the yeast Saccharomyces cerevisiae. Genetics 2012, 190:317-349.

7. Graeve M, Janssen D: Improved separation and quantification of neutral and polar lipid classes by HPLC-ELSD using a monolithic silica phase: application to exceptional marine lipids. J Chromatogr B Analyt Technol Biomed Life Sci 2009, 877:1815-1819.

8. Guerfal M, Ryckaert S, Jacobs PP, Ameloot P, Van Craenenbroeck K, Derycke R, Callewaert N: The HAC1 gene from Pichia pastoris: characterization and effect of its overexpression on the production of secreted, surface displayed and membrane proteins. Microb Cell Fact 2010, 9:49.

9. Tange Y, Hirata A, Niwa O: An evolutionarily conserved fission yeast protein, Ned1, implicated in normal nuclear morphology and chromosome stability, interacts with Dis3, Pim1/RCC1 and an essential nucleoporin. J Cell Sci 2002, 115(22):4375-4385.

10. Han GS, Wu WI, Carman GM: The Saccharomyces cerevisiae Lipin homolog is a Mg2 +-dependent phosphatidate phosphatase enzyme. J Biol Chem 2006, 281(14):9210-9218

11. Fickers P, Le Dall MT, Gaillardin C, Thonart P, Nicaud JM: New disruption cassettes for rapid gene disruption and marker rescue in the yeast Yarrowia lipolytica. J Microbiol Methods 2003, 55:727-737.

12. Fraser NJ: Expression and functional purification of a glycosylation deficient version of the human adenosine 2a receptor for structural studies. Protein Expr Purif 2006, 49:129-137.

13. Sidrauski $C$, Walter $P$ : The transmembrane kinase Ire $1 p$ is a site-specific endonuclease that initiates mRNA splicing in the unfolded protein response. Cell 1997, 90:1031-1039.

14. Oh MH, Cheon SA, Kang HA, Kim JY: Functional characterization of the unconventional splicing of Yarrowia lipolytica HAC1 mRNA induced by unfolded protein response. Yeast 2010, 27:443-452.

15. Rasmussen SG, Choi HJ, Rosenbaum DM, Kobilka TS, Thian FS, Edwards PC, Burghammer M, Ratnala VR, Sanishvili R, Fischetti RF, Schertler GF, Weis WI, Kobilka BK: Crystal structure of the human beta2 adrenergic G-proteincoupled receptor. Nature 2007, 450:383-387.

16. Rasmussen SG, DeVree BT, Zou Y, Kruse AC, Chung KY, Kobilka TS, Thian FS, Chae PS, Pardon E, Calinski D, Mathiesen JM, Shah ST, Lyons JA, Caffrey M, Gellman SH, Steyaert J, Skiniotis G, Weis WI, Sunahara RK, Kobilka BK: Crystal structure of the beta2 adrenergic receptor-Gs protein complex. Nature 2011, 477:549-555.

17. Fakas S, Qiu Y, Dixon JL, Han GS, Ruggles KV, Garbarino J, Sturley SL, Carman GM: Phosphatidate phosphatase activity plays key role in protection against fatty acid-induced toxicity in yeast. J Biol Chem 2011, 286:29074-29085.

18. Rockenfeller $\mathrm{P}$, Ring J, Muschett V, Beranek A, Buettner S, Carmona-Gutierrez D, Eisenberg T, Khoury C, Rechberger G, Kohlwein SD, Kroemer G, Madeo F: Fatty acids trigger mitochondrion-dependent necrosis. Cell Cycle 2010, 9(14):2836-2842

19. Blazeck J, Reed B, Garg R, Gerstner R, Pan A, Agarwala V, Alper HS Generalizing a hybrid synthetic promoter approach in Yarrowia lipolytica. Appl Microbiol Biotechnol 2013, 97(7):3037-3052.

20. Blazeck J, Liu L, Redden H, Alper H: Tuning gene expression in Yarrowia lipolytica by a hybrid promoter approach. Appl Environ Microbiol 2011, 77(22):7905-7914. 
21. Csaki LS, Reue K: Lipins: multifunctional lipid metabolism proteins. Annu Rev Nutr 2010, 30:257-272.

22. Gaj T, Gersbach CA, Barbas CF 3rd: ZFN, TALEN, and CRISPR/Cas-based methods for genome engineering. Trends Biotechnol 2013, 31(7):397-405.

23. Echeverri CJ, Perrimon N: High-throughput RNAi screening in cultured cells: a user's guide. Nat Rev Genet 2006, 7(5):373-384.

24. Hartner FS, Ruth C, Langenegger D, Johnson SN, Hyka P, Lin-Cereghino GP Lin-Cereghino J, Kovar K, Cregg JM, Glieder A: Promoter library designed for fine-tuned gene expression in Pichia pastoris. Nucleic Acids Res 2008, 36(12):e76.

25. De Pourcq K, Vervecken W, Dewerte I, Valevska A, Van Hecke A, Callewaert N: Engineering the yeast Yarrowia lipolytica for the production of therapeutic proteins homogeneously glycosylated with Man8GlcNAc2 and Man5GIcNAc2. Microb Cell Fact 2012, 11:53.

26. Barth G, Gaillardin C: Physiology and genetics of the dimorphic fungus Yarrowia lipolytica. FEMS Microbiol Rev 1997, 19:219-237.

27. Baharaeen S, Vishniac HS: A fixation method for visualization of yeas ultrastructure in the electron microscope. Mycopathologia 1982, 77:19-22.

28. Hellemans J, Mortier G, De Paepe A, Speleman F, Vandesompele J: qBase relative quantification framework and software for management and automated analysis of real-time quantitative PCR data. Genome Biol 2007, 8:R19.

29. Folch J, Lees M, Sloane Stanley GH: A simple method for the isolation and purification of total lipids from animal tissues. J Biol Chem 1957, 226:497-509.

doi:10.1186/1475-2859-12-122

Cite this article as: Guerfal et al:: Enhanced membrane protein expression by engineering increased intracellular membrane production. Microbial Cell Factories 2013 12:122.

\section{Submit your next manuscript to BioMed Central and take full advantage of:}

- Convenient online submission

- Thorough peer review

- No space constraints or color figure charges

- Immediate publication on acceptance

- Inclusion in PubMed, CAS, Scopus and Google Scholar

- Research which is freely available for redistribution 